\title{
A New Theory for Human Social Development
}

\author{
Herbert Loveless ${ }^{1}$ \\ ${ }^{1}$ Affiliation not available
}

February 24, 2021

\begin{abstract}
A new theory for the social development of man is proposed based on the skeletal similarity between Ardi (Ardipithecus ramidus). It has been the standard theory to model the earliest social development of human ancestors on the chimpanzee because this species, along with the bonobo, is closest to man genetically. However, the chimpanzee, bonobo an gorilla are all knuckle walkers with "hunched over, constricted" skeletons. Both Ardi and the orangutan have extended skeletons. This suggests that the two species had similar forms of locomotion and that Ardi spent virtually its entire life in the forest canopy as the orangutan does, and there for that Ardi was pre-evolved for bipedal locomotion. It also suggests that Ardi might have exhibited social behavior similar to what the orangutan exhibits both in nature and when raised in rescue centers.
\end{abstract}

A New Theory for Human Social Development

Herbert Loveless MD

It has been the standard theory to model the earliest social development of human ancestors on the chimpanzee because this species, along with the bonobo, is closest to man genetically. However, the anatomy of the chimpanzee/bonobo along with the gorilla is markedly different than that of the 4.4-million-year-old (myo) human ancestor, Ardi (Ardipithecus ramidus). $(1,2)$ This is because the former three are all knuckle walkers adapted to moving on the forest floor on all four limbs as well as climbing trees. They have a "constricted, hunched over" skeleton with the hip and knee joints flexed in the neutral position. Ardi, in contrast, has an elongated skeleton that some believed allowed for bipedal locomotion.

The living ape with the skeleton that most closely resembles Ardi's is the orangutan. This species has an elongated skeleton that enables maximum reach by its upper limbs for overhead branches while its lower limbs have a narrow gate that enables best balance while walking "foot over foot" on branches and vines. This close ness in anatomy suggests a similarity in behavior.

In contrast to the chimpanzee, bonobo and gorilla that spend a large portion if not the majority of there time on the forest floor, the orangutan spends virtually its entire life in the forest canopy. They have a unique method for moving across a gap between trees. They wave a branch quickly back and forth, eventually using it as a "spring lever" to obtain momentum to move between a gap in between trees. (3)

There is one dominant male in a territory with wide facial flares. The orangutans live dispersed throughout this territory. The female raises one offspring at a time. The offspring stays with its mother for about eight years. This is a longer maturation period that the gorilla, chimpanzee and bonobo. During this maturation period, the offspring learns the intricate task of weaving a sleeping platform. This task is far more intricate than learning how to crack nuts with a rock, or other tasks learned by the other three great apes. The dominant male maintains contact with others in its territory by vocalizations. There is almost never any physical contact between orangutans other than for sex.

Because of loss of their mothers caused by deforestation, rescue centers are raising very young, rescued orangutans in age groups. During this period, the orangutans learn the skills necessary for a return to the 
forest. Each age group is feed separately on a communal feeding platform. During these periods, the young orangutans exhibit social behavior reminiscent of age-appropriate human children.

Clearly one must be cautious in how far to apply the behavior of the orangutan to Ardi and other early human ancestors. It is possible that only the locomotion on a limb, and not that going between gaps as well as having a single dominant male and any other social behavior. But the use of the orangutan as a model suggests that because "upright" locomotion in the forest canopy, Ardi was pre-evolved for bipedal locomotion as the forest gradually was replaced by savanna.

There is also a possibility that any social organization facilitated by this lifestyle also had Ardi pre-evolved for formation of social behavior that served as the basis for the further evolution of social behavior on the savanna. It also suggests that necessity to learn intricate task(s) pre-evolved Ardi's descendants for the evolution neurologic systems that enabled more complex behavior than exhibited by the gorilla, chimpanzee and bonobo.

1. Gibbons, Ann. Science Vol 326, Issue 5926:1598-1599. Dec. 18, 2009.

2. Lovejoy, C Owen. Science 326, Issue 5949:74-74e8. Oct 2, 2009.

3. Thrope, Susannah KS, Holder, Roger and Compton, Robin H. PNAS 106(31):12646-12651. Aug. 4, 2009 . 